\title{
Stellar Dynamics and the 3D Structure of Bars
}

\author{
D. Pfenniger
}

\author{
Geneva Observatory, CH-1290 Sauverny, Switzerland
}

\begin{abstract}
Recent observational constraints restrict the strict applicability of stellar dynamics in spirals to a few rotation periods. However, stellar dynamics concepts such as periodic orbits are invaluable for understanding the various dynamical processes occurring during much more periods. A distinction of two instability types in stellar systems is pointed out, the first one being well illustrated by the bar instability, and the second one by the bar bending instability. In bars the third dimension brings essential dynamical effects which modify the views about the history of bulges and the spiral secular evolution. Bars may grow, bend, thicken, and dissolve into spheroidal bulges, and spirals may evolve along the Hubble sequence in the sense $\mathrm{Sd} \rightarrow \mathrm{Sa}$. This leads to a much more dynamical picture of isolated galaxies than imagined before.
\end{abstract}

\section{Applicability of Stellar Dynamics in Spirals}

Recent developments about spirals bring a quite different picture about their physical state. The discovery of their large far-infrared (FIR) flux by IRAS and COBE, comparable or sometimes superior to the optical one, and consistent with the evidences that their optical parts are semi-transparent, means that a substantial fraction of the stellar light is thermalized, and re-emitted by dust in the FIR. This is not negligible with respect to the the typical power that largescale dynamical processes (spiral arms, bars) can exchange, the gravitational power (ratio of the gravitational energy to the dynamical time). For a system in near virial equilibrium, $v^{2}=G M / R$, we have $L_{\text {grav }} \approx\left(G M^{2} / R\right) /(R / v)=$ $v^{5} / G$. Replacing $v$ by the typical rotation speeds of spirals we find powers surprisingly close to the the galaxy luminosities (e.g. $10^{44} \mathrm{erg} \mathrm{s}^{-1}$ for the Milky Way). Since the IR Tully-Fisher relation $\left(L_{\mathbf{H}} \propto v^{4-5}\right)$ is nearly parallel to this equation, the match is close along the whole spiral sequence (for more references on the subject see Pfenniger 1992).

This coincidence supports the proposition that a feedback mechanism relates both dynamical instabilities and stellar activity. Essential is that although a stellar population pours out energy over several Gyr, the first Myr of a starburst is a quicker cooperative and intense reaction with respect to the dynamical time $\tau_{\text {dyn }}$. The idea of a feedback mechanism between dynamics and star formation has been proposed several times (e.g. Quirk 1972; Kennicutt 1989). With a light thermalization power of the order of the gravitational power, we just need to convert at an intermediate stage (between light production and thermalization) a sizable fraction of this power into mechanical energy. It is quite obvious 
in images of HII regions and starbursts that a substantial mass of surrounding gas is set into coherent motion by the massive star heating; this yields ultimately HI holes and superbubbles. If a fraction $\gamma$ of the stellar power is converted into mechanical power, the time-scale to change significantly the global binding energy of the whole galaxy is $\tau_{\text {dyn }} / \gamma$. If $0.02<\gamma<1$ then the evolution time-scale associated with stellar activity is shorter than the galaxy age; the galaxy as a whole must depend on the stellar energy output. $\gamma$ is not well known but is estimated to be in the range $\gamma \approx 0.1$ in starbursts (Leitherer \& Heckman 1995).

Such simple facts change fundamentally the way to see spirals. In the early days of galactic dynamics, galaxies were viewed as essentially transparent collections of stars, therefore the stellar energy output could be discounted, exactly as the huge supernova neutrino flux. Also the large scale dynamical instabilities such as bars were not understood, only the slow 2-body relaxation was considered as a factor of evolution, then the dimension and shape of galaxies had to be viewed as determined by the initial conditions of formation. The subsequent evolution was viewed as so slow that stellar dynamics concepts could be used for several Gyr. This led to absorb all the dynamical effects in a rigid potential in many models, such as the stellar population synthesis ones.

The conjunction of new elements on the energetics of spirals forces us to modify the way to use stellar dynamics. Which of the concepts such as relaxation, integral of motion, etc., are still relevant and what are their new limits of applicability? If we take the Milky Way as a template of the spirals, from observational data it is clear that the virial gross equilibrium, at least in the optical disk, must be consistent with a dominance of the bulk kinetic energy $E_{\text {kin }}$ balancing gravitational energy $E_{\text {grav }}$ :

$$
\frac{1}{2} \ddot{I}=\underbrace{-3}_{\sim 4000 \mathrm{eV} \mathrm{cm}} \times V=\underbrace{2 E_{\text {kin }}}_{>\sim 1000 \mathrm{eV} \mathrm{cm}^{-3} \times V}+\underbrace{E_{\text {grav }} \mid}_{\sim 10 \mathrm{eV} \mathrm{cm}}-\underbrace{3 P_{\text {int }}}_{<1 \mathrm{eV} \mathrm{cm}^{-3}} V,
$$

where $I$ is the moment of inertia inside the considered volume $V$. The inner pressure $P_{\text {int }}$ due to all the ISM components (gas, cosmic rays, etc.) is a much too small energy reservoir to play any important role in the virial equilibrium. The outer pressure $P_{\text {ext }}$ due to intergalactic gas and radiation is even more negligible. The only known large enough negative contribution to the virial balance susceptible to compensate the large positive contribution of $E_{\mathbf{k i n}}$ is gravitational, although the detected mass is still insufficient. Thus dark matter must be invoked, particularly in the outer disks of spirals.

Now the equilibrium is certainly imperfect as we have seen above with the large visible and IR fluxes $\left(\sim 10^{44} \mathrm{erg} \mathrm{s}^{-1}\right)$ through the interstellar medium. The quasi-static evolution of systems in near virial equilibrium is to first order:

$$
\ddot{I}(t)=\underbrace{\ddot{I}(0)}_{\sim 0}+t \frac{d \ddot{I}(0)}{d t}+\mathcal{O}\left(t^{2}\right) \approx 2 t\left[2 \dot{E}_{\mathrm{kin}}+\dot{E}_{\mathrm{grav}}+3 \dot{P}_{\mathrm{int}} V-3 \dot{P}_{\mathrm{ext}} V\right]+\mathcal{O}\left(t^{2}\right)
$$

Hence, while an equilibrium needs similar interacting energies, the slowest quasistatic evolution needs also similar interacting powers cancelling each other. This is precisely what is suggested by FIR data on spirals.

Thus over time-scales of the order of $1-100 \mathrm{Myr}$ a spiral may be considered as in equilibrium at the largest scale because then the shortest relevant evolution 
time-scale is the longer dynamical time. However, at smaller scale $(\sim 1 \mathrm{kpc})$ the local power imbalance may be large due to either the energy output by massive stars, or the fast radiative cooling leading to dense molecular clouds.

Over time-scales of the order of 0.1 - 10 Gyr the heating resulting from stellar activity and gas cooling in average must mostly cancel. The feedback mechanism regulating star formation at a constant average level via dynamics is essential, otherwise one would expect the spiral as a whole either to explode or to collapse rapidly. A tight feedback requires a minimum galaxy size in order to reduce the fluctuations; for too small galaxies such as dwarfs, a couple of OB stars in excess is already significant to disrupt the galaxy: there one may find a reason for a minimum size for star forming systems looking like spirals ${ }^{1}$.

Over longer time-scales, say $1-10 \mathrm{Gyr}$, the gas consumption by a sustained star formation is substantial, and eventually the feedback weakens. Furthermore, other dynamical processes, such as a bar instability or mergers, can become relevant and may modify the conditions of star formation.

After such considerations the initial conditions of galaxies look secondary for the present state. More important is the galactic micro-physics: star formation and the ISM properties. Physically this is a much more comfortable situation because nowadays we expect rather chaotic initial conditions of formation as more realistic than the ordered collapses envisioned for decades. In order to obtain galaxies with systematic properties the information must be encoded, as for stars, within the matter instead of the initial conditions.

\section{Stellar Dynamics as a First Order Tool}

Consequently, stellar dynamics seems now clearly incomplete for the understanding of spirals over $10-15 \mathrm{Gyr}$. However, for shorter time-scales like a few rotational periods, since the dominating energies are kinetic and gravitational, we can indeed approximate the full Boltzmann equation (as applied either to stars or molecules)

$$
\frac{\partial f}{\partial t}+\vec{v} \cdot \vec{\nabla} f-\vec{\nabla} \Phi \cdot \frac{\partial f}{\partial \vec{v}}=\left(\frac{\partial f}{\partial t}\right)_{\text {coll }},
$$

by neglecting the right-hand side (rhs) collisional term. Stellar dynamics can thus still be applied, but over time-scales much shorter than believed earlier.

For the optical parts of galaxies where the stellar mass dominates, the meanfree path of stars is large and furthermore often collective effects can be neglected because the kinetic energy in non-systematic motion is large enough to yield not too small Jeans' lengths. In sufficiently hot systems collective effects are small at scales smaller than the Jeans' length, thus the potential is replaced by a mean gravitational potential $\Phi$. In such a mean-field approximation galactic dynamics reduces to describing the possible orbits in the potential $\Phi$,

$$
\ddot{\vec{x}}=-\vec{\nabla} \Phi(\vec{x}) \text {. }
$$

\footnotetext{
${ }^{1}$ An upper spiral velocity weakly dependent on their mass follows from the maximum time-scale $\tau_{\max }$ to consume all the nuclear energy in galaxies with the feedback $L \approx v^{5} / G$. Then $\tau_{\max } \ll$ $0.008 G M c^{2} / v^{5}$, so $v<\left(0.008 G M c^{2} / 12 \mathrm{Gyr}\right)^{1 / 5} \approx 500_{-200}^{+300} \mathrm{~km} \mathrm{~s}^{-1}$ for $M=10^{11 \pm 1} M_{\odot}$.
} 
This is a problem belonging to the well studied Hamiltonian mechanics. Let us recall a few elementary general properties of such systems (e.g. Arnold 1989), useful to know before analyzing the phase space structure of galactic potentials.

The first important concept is the one of orbit, to distinguish from the one of trajectory. An orbit is the whole subset of phase-space generated over an infinite time in the past and in the future by an initial condition in a dynamical system such as Eq.(4). A trajectory is the subset of phase-space visited over a finite time by some initial point. By construction an orbit is an invariant subset of phase space by the motion, because all the points along an orbit generate trajectories within it. Orbits are thus the most fundamental invariant blocks with which we can build equilibrium models of stellar systems. Stellar systems may be seen as made of orbits instead of stars.

There are several different types of orbits in $3 D$ stellar systems which are characterized by different dimensions $d$ in the 6 -dimensional phase space. Either they have $d=0$ if they don't move, they are fixed points, or at least $d=1$ if they move, and at most $d=5$ in equilibrium systems due to the energy integral. The only orbits with non-vanishing phase space volumes are usually the quasiperiodic orbits with $d=3$ and the chaotic orbits with fractal, non-integer $d$. The neighborhood of fixed points and periodic orbits is either stable or unstable depending on whether or not most of the neighboring trajectories remain close. In case of instability the neighborhood is mostly made of chaotic orbits. These properties are summarized as follows:

\begin{tabular}{|c|c|c|c|}
\hline Orbit type & Dimension $d$ & $\begin{array}{l}\text { Neighborhood } \\
\text { if stable }\end{array}$ & $\begin{array}{l}\text { Neighborhood } \\
\text { if unstable }\end{array}$ \\
\hline & 0 & periodic orbits & $\operatorname{chac}$ \\
\hline peri & 1 & Deriodic & chaotic orbits \\
\hline quasi-peri & 2, or 3 & quasi-periodic orb. & \\
\hline chaotic & fractal: $1<d \leq 5$ & - & chaotic orbits \\
\hline
\end{tabular}

The chaotic orbit class is the class of the orbits not classifiable in the other classes (like the 'peculiar' galaxies in the Hubble classification). It includes different types of orbits which could be further sub-classified according to their multifractal spectrum and fractal dimension.

As understood long ago by Poincaré, periodic orbits are certainly, after the few fixed points, the most important class of orbits, not because many stars are on them, but because when stable they summarize the surrounding phasespace. The stable periodic orbits are always surrounded by concentric quasiperiodic orbits, or tori. Those tori are much more numerous. This structure of concentric tori surrounding the periodic orbits in Hamiltonian systems is the fundamental property allowing one to conceptualize phase-space by its stable periodic orbits. This property is still insufficiently appreciated, even by some dynamicists, motivating these elementary reminders.

Among the robust properties of orbits we note: 1) the shapes of the main periodic orbits depend only on the potential symmetries, therefore we do not need a precise description of the galaxy to know its orbital structure, 2) the periodic orbits are insensitive to low spatial frequency perturbations, so moderate low frequency symmetry breaking (such as a bar bending out of the plane) does not change much the orbital structure, it only deforms it slightly, and 3 ) the stable periodic orbits become attractors by a weak dissipative perturbation. A 
small dissipation in fact condenses the trajectories of the system toward its most fundamental periodic orbits.

However, periodic orbits are fragile against high spatial frequency perturbations anywhere along their path. For example, a small accumulation of mass just at the galaxy center may profoundly change the stability of the radial periodic orbits crossing the center, and so the structure of the surrounding phase space.

If we consider now all the neglected effects that should be taken into account to reflect all the complexity of a real spiral, such as gas dynamics and star formation, among all the possible structures of a pure stellar dynamical representation the main stable periodic orbits are certainly the ones that survive the longest and are the best suited to describe evolution features. They become inevitable concepts to understand complex non-linear dynamical processes. This reality can be experienced by those dealing with the complexity of $N$-body simulations, also including gas. It is quite obvious that, for example, when we think about a rotating disk we make a "thought economy" by first considering only its circular orbits, even if no stars follow exactly such orbits.

\section{Phase-Space Structure of Barred Galaxies}

The main dynamical feature of a barred galaxy is shaped by its only general isolating integral, the Jacobi integral, also called the "energy" in a rotating frame of reference. The Hamiltonian in the rotating frame of the bar reads

$$
H=\frac{1}{2}\left(p_{x}^{2}+p_{y}^{2}+p_{z}^{2}\right)+\Phi(x, y, z)-\Omega_{\mathrm{p}}\left(x p_{y}-y p_{x}\right),
$$

where the momenta $p_{x}=\dot{x}-\Omega_{\mathrm{p}} y, p_{y}=\dot{y}+\Omega_{\mathrm{p}} x$, and $p_{z}=\dot{z}$ are the velocity components in the instantaneous parallel inertial frame. The zero-velocity surface (ZVS) in the rotating frame

$$
H_{0}=\Phi(x, y, z)-\frac{1}{2} \Omega_{\mathrm{p}}^{2}\left(x^{2}+y^{2}\right)
$$

bounds motion in space at low "energy" inside a football shape (nearly like a spheroid) inside corotation. Higher "energy" particles inside the bar can escape first through tunnels in the ZVS near the end of the bar, and then through the whole corotation circle at still higher "energy". Four of the five fixed points, the Lagrangian points, are located at the corotation.

The closest simple analytical model of a barred galaxy is an axisymmetric disk, in which the basic unperturbed orbits are the circular orbits in the principal plane. The three orbital frequencies in the plane are the rotation frequency $\Omega$, the radial and vertical epicyclic frequencies $\kappa$ and $\nu$. These frequencies squared depend only on the potential local derivatives at $z=0$ (see Binney \& Tremaine 1987, p. 121). Circular orbits are then stable if $\Omega^{2}>0, \kappa^{2}>0$, and $\nu^{2}>0$.

If we consider the bar as a perturbation of the circular case, the circular orbits are perturbed by the non-axisymmetric potential rotating at the frequency $\Omega_{\mathrm{p}}$. The resulting transverse deviations $\xi$ from the circular orbits (either radial or vertical) can be described by the Hill's equation, that is the equation of a harmonic oscillator the natural frequency $\omega_{0}$ of which is periodically modulated:

$$
\ddot{\xi}+\omega_{0}^{2}[1+\epsilon(t)] \xi=0,
$$


with $\epsilon(t)$ a small periodic function of frequency $\omega_{\text {per }}$. A general theorem (see e.g. Arnold 1989) gives the parametric resonances conditions for arbitrarily small modulation $\epsilon(t)$ :

$$
\frac{\omega_{0}}{\omega_{\text {per }}}= \pm \frac{k}{2}, \quad k=0,1,2, \ldots \infty
$$

With this theorem we can derive elegantly the resonance conditions, because we do not need to know the precise form $\epsilon$ of the bar perturbation, but only the perturbing frequency $\omega_{\text {per. }}$. To determine the resonance widths one does need however to know the particular form of $\epsilon$.

The radial and vertical epicyclic frequencies $\kappa$ and $\nu$ are the natural oscillation frequencies around the circular orbits, and the bar perturbing frequency is $2\left(\Omega \mp \Omega_{\mathrm{p}}\right)$ for direct/retrograde circular orbits. The factor 2 comes from the bi-symmetry of the bar (the "number of arms"). Then we obtain the classical resonances when

$$
\frac{\kappa}{\Omega \mp \Omega_{\mathrm{p}}}= \pm m, \quad \frac{\nu}{\Omega \mp \Omega_{\mathrm{p}}}= \pm n, \quad m, n=0,1,2, \ldots \infty .
$$

The main direct resonances encountered in barred galaxies occur for $m=n=$ $\infty$ (corotation), $m=n=2$ (radial and vertical inner Lindblad resonances), $m=-2$ (radial outer Lindblad resonance), and $m=n=4$ (radial and vertical ultra-harmonic resonances). Around corotation we have the piling up of an infinity of higher order resonances. The main purpose of recalling these simple considerations is to stress that the treatments of radial and vertical resonances are the same, there is no ground to neglect the vertical resonances, particularly because bars extend in the central regions of the galaxies which are not much thinner than large.

In addition, the knowledge of the resonances gives the approximate shapes of the nearby orbits. For having elongated oval orbits we always expect that the conditions are close to have a 2/1 Lindblad resonance, because an oval is a $2 / 1$ perturbation of a circle. Rectangular shapes are associated with $4 / 1$ resonances, etc. This applies in the vertical direction too, a $2 / 1$ resonance is associated with $2 / 1$ vertical oscillations out of the plane, etc. Once the typical resonances in barred galaxies are known, it is simple to guess what the main periodic orbits look like. Detailed numerical calculations of orbits can help in quantifying precisely the orbit positions and shapes, and also in finding higher order periodic orbits. Numerous works have been made in this area allowing to grasp the main general features of the bar dynamics (see references, e.g., in the review of Sellwood \& Wilkinson 1993). The general properties are well understood in a descriptive way by several numerical calculation works. Although the main periodic orbit families in the plane and in 3D, including their shape, are well known by now, there is still no simple but realistic analytical model of galactic bars.

Exactly similar considerations to circular orbits apply for radial periodic orbits. A slight complication is that for the $z$-axis orbits in a rotating bar the two transverse frequencies are coupled by rotation, which leads to a generalization of the Hill's equation and new possibilities of parametric resonances via "complex instability" (Pfenniger 1987). But in non-rotating bars, Hill's equation (7) applies for each transverse component of the three types of axial orbits, the 
perturbing frequency being the oscillation frequency of the radial orbit. Then a 2/1 resonance leads to banana shaped orbits (shaping the neighboring phase space) which favors a bending instability in non-rotating ellipsoidal systems too.

For oblate axisymmetric disks with positive density (constraining the frequencies by $2 \Omega^{2}<\kappa^{2}+\nu^{2}$, and $\Omega=c \nu$, where $c<1$ is the potential axis ratio) a central mass concentration always produces low order radial and vertical resonances. Now as long as the potential remains axisymmetric the width of the resonance is zero, and these resonances are ineffective. But as soon as a triaxial deformation exists, the resonance widths grow which fosters chaotic motion. Then diffusion in the vertical direction is natural near the center of a galaxy, especially if slow dissipative processes accrete mass near the bottom of the potential. Then the idea that a bulge may naturally grow in a barred potential is dynamically justified (Pfenniger 1984, 1985; Pfenniger \& Norman 1990).

The dissolution of a bar by a growing central mass concentration is an example of a possible qualitative prediction allowed by knowing the periodic orbits of bars (Hasan \& Norman 1990; Pfenniger \& Norman 1990; Hasan et al. 1993). Since the radial and vertical ILR radii increase rapidly when the central mass concentration increases $R_{\mathrm{ILR}} \propto M_{\mathrm{Cm}}^{2.8}$, and the elongated orbits supporting the bar are replaced by orbits perpendicular to the bar inside the growing ILR radius, the elongated shape of the bar is rapidly no longer compatible with the growing central mass concentration. Orbit calculations show that around $5-15 \%$ percent of the disk mass as a central mass concentration should be sufficient to destroy the bar. Since the vertical ILR is typically close to the radial ILR, one expects an extended 3D diffusion of the initial central part of the disk.

To conclude this Section, simple periodic orbit considerations show that barred galaxies have numerous resonances and associated chaotic zones from which we can expect instabilities. For studying these we need however techniques able to take self-gravitation into account, such as $N$-body methods.

\section{Types of Instabilities in Collisionless Gravitating Systems}

Many works have considered the problem of self-consistent stability with the linearized Collisionless Boltzmann equation. Here we just point out how this method may help in distinguishing two different sources of instabilities in real stellar systems, simultaneously clarifying the limitations of the method.

Suppose we know a solution $f_{0}, \Phi_{0}\left(\rho_{0}=\int f_{0} d^{3} v\right)$ of the Collisionless Boltzmann and Poisson equations. Then, as usual, we want to describe the evolution of small perturbations $\delta f, \delta \Phi$ around the assumed known solution $f_{0}$, $\Phi_{0}$ in the linear approximation. Linearizing the equations we obtain:

$$
\begin{aligned}
& \text { orbital behavior, mixing of } \delta f \quad \text { source of } \delta f \\
& \overbrace{\frac{\partial \delta f}{\partial t}+\vec{v} \cdot \vec{\nabla} \delta f-\vec{\nabla} \Phi_{0} \cdot \frac{\partial \delta f}{\partial \vec{v}}}^{\mathrm{D} \delta f} \frac{\mathrm{D} t}{\mathrm{D} t}=\overbrace{\vec{\nabla} \delta \Phi \cdot \frac{\partial f_{0}}{\partial \vec{v}}}^{,}, \\
& \vec{\nabla} \cdot \vec{\nabla} \delta \Phi=4 \pi G \delta \rho
\end{aligned}
$$

As set, Eq.(10) shows that the variations $\delta f$ depend on two simple effects.

First, the rhs of Eq.(10) shows that to modify the density of a volume element moving around a given unperturbed orbit, a gradient of $f_{0}$ in the velocity 
space is required. Furthermore, only the force fluctuation component parallel to the velocity gradient contributes. Large density gradients in velocity space favor instabilities parallel to the gradient (so radial in cold rotating disks).

The second factor of effective instability is a little more subtle. The lefthand side (lhs) term, $D \delta f / D t$, describes the Lagrangian derivative in phase space of a fluctuating element along an unperturbed orbit. So, even if the rhs vanishes, or is very small, the form of a compact element may be rapidly modified into a threaded structure, the fineness of which becomes smaller than allowed by the various approximations. This occurs precisely around unstable orbits in the unperturbed system, such as chaotic orbits. This sensitive dependence means that the neglected terms (higher order terms, collision and dissipative terms) become rapidly relevant for the real system ${ }^{2}$.

Thus in stellar systems which contain chaotic regions of phase space, so typically around resonances, an initial compact volume element of $\delta f$ is rapidly distorted into very intermingled shapes, the slightest perturbation is exponentially amplified, and non-linear considerations are very soon necessary. In such a situation it is more natural to ascribe the resulting instability not to the rhs source term, but to the lhs phase space structure. On the other hand, when the orbital motion is regular, a compact initial volume element remains compact much longer, the lhs is well-behaved and a growing mode can only be attributed to the rhs term. In such cases the instability can be ascribed to the full distribution function $f_{0}$. Note that analytical models have usually a regular phase space, so the problem appears preferentially in numerical models not biased against chaos.

In summary, in order to progress in the understanding of stellar systems and their instabilities we can divide their analysis in two distinct parts:

1. The understanding of the phase-space structure (i.e. the orbits) of the potential $\Phi_{0}$ independently of the velocity structure of $f_{0}$. The resonance regions and the chaotic zones are then susceptible to seed collective instabilities and non-linear effects not describable with a linear theory.

2. The understanding of the specific self-gravity effects associated with particular $f_{0}$ 's consistent with a given $\Phi_{0}$. One may generally expect that sufficiently cold distribution functions should produce instabilities not directly related to the previous aspect. For a regular phase-space the linear mode analysis should work much better.

As illustration, in spirals the bar instability comes mainly from a too cold $f_{0}$, because phase space surrounding stable circular orbits is regular, while the bending instability leading to peanut-shaped bars, discussed below, is mainly due to a $2 / 1$ vertical resonance and is little dependent on the velocity structure of $f_{0}$.

\footnotetext{
${ }^{2}$ The same occurs in many other problems: when the linear operator $A$ of the problem $A x=B$ has widely different singular values then the problem is ill-posed and the solution $x$ is ill-defined.
} 


\section{Smoothness Assumption}

An assumption which is most of the time not discussed is that $f$ should be a differentiable function. In ordinary gases with molecules having short range interactions and frequent collisions, any irregular distribution is rapidly smoothed out locally by the "molecular chaos". The smoothing principle comes then from the short relaxation time and the lack of long-range interactions, which allows a fast local decorrelation and homogenization of the particles.

In collisionless gravitating systems this rapid smoothing is far from being obvious and demonstrated. As soon as instabilities occur, the long range of gravitation correlates the fluctuating part $\delta f$ much more than in ordinary gas, and since we lack of a smoothing principle, no good reason other than commodity allows to assume that a differentiable $\delta f$ is a valid assumption allowing its use in the variational differential equation.

In fact numerical experiments indicate rapid limitations of the linear theory. For example Toomre \& Kalnajs (1991) have simulated a small portion of a self-gravitating flat disk which is maintained slightly unstable. The results show clearly that long range correlations are rapidly created and do persist; a fractal scale invariant and dynamical state follows. The linearization approach is of no use for describing such states. Another well documented case of gravitational instability occurs in the numerous simulations of expanding universes. The runs develop usually non-linear fractal structures which cannot be derived from a linear study. In such a situation, analogous to turbulence in fluids, the system has a sensitive dependence on small-scale effects and perturbations. The collisional as well as weak dissipative effects can be crucial. Therefore we should be cautious about drawing conclusions without a thorough non-linear analysis. $N$ body methods are presently essential to study non-linearities with the advantage that they include non-vanishing collisional effects.

\section{Self-Consistent 3D Barred Galaxy Models}

In the early 80 's it was generalized from a single peculiar edge-on S0 galaxy with a small(!) bulge (NGC 4762) that bars are generally flat $(a / c \sim 10)$ (see Kormendy 1982). Theory says such flat bars are implausible to maintain for a long time since they imply highly anisotropic velocity distribution, a strong velocity gradient of $f_{0}$, and strong vertical resonances from the bar potential.

In fact in 3D $N$-body simulations most of the initially flat bars thicken rapidly and are subject to bending instabilities transverse to the plane (Friedli \& Pfenniger 1990; Pfenniger \& Friedli 1991 (PF91); Raha et al. 1991). The end-result of these bending instabilities are box- or peanut-shaped bars (Miller \& Smith 1979): stable structures over several Gyr resembling much the observed peanut-shaped bulges according to viewing angle of the bar (Combes \& Sanders 1981; Combes et al. 1990; see also references in Merrifield, these proceedings). The peanut-shaped bars look round when viewed end-on.

While Raha et al. have identified this instability with the "fire-hose instability" (the out of the plane instability of an infinite homogeneous thin sheet), the orbital analysis in PF91 shows clearly that the fire-hose instability picture is a too rough analogy to predict the bending instability main characteristics 
such as its size, the principal mode of bending, and the instability threshold. The detailed analysis of the $N$-body bar run in PF91 (see also Pfenniger 1990) greatly improves the understanding of the instability by considering not only 1) its simple morphological and kinematic description, but also 2) its potential resonances, which give a first idea on the orbits, further 3 ) its periodic and other orbits at different times, and then 4) its distribution function changes. Finally the whole non-linear evolution of the ensemble can be much better understood.

Non-linearities are essential all along the phenomenon: the instability starts near the vertical $2 / 1$ resonance, so is associated with this resonance, it chooses to bend up or down randomly from the fluctuations, with precisely a $2 / 1$ banana shaped mode, then it symmetrizes rapidly its vertical profile. The instability saturates around $2 / 2 / 1$ stable periodic orbits, which pre-exist, accompany and survive the instability, explaining the final peanut shape. As the instability proceeds, the vertical $2 / 1$ resonance sweeps the bar particles from low to high "energy", allowing most of them to leave the plane up to heights and distances allowed by the ZVS. Thus, the bending instability is a nice example of a gravitational instability little dependent on the velocity space structure of $f_{0}$, it occurs also in bars initially far from being flat. Clearly, pure orbital considerations, lacking of the self-gravity, are insufficient to predict firmly the bending and time evolution. But a linear mode analysis such as in Merritt \& Sellwood (1994) is unable to describe the whole phenomenon too ${ }^{3}$. It is only the association of the two approaches together with the $N$-body technique which allows a detailed understanding of the entire process.

As noted in Section 3, the knowledge of the orbital structure in bars is also useful for predicting conditions of their destruction. This can be tested with $N$-body runs, which confirm that indeed a mass accumulation within the original ILR changes the orbital structure to an extent that elongated bars are no longer possible, but only $1-3 \%$ of additional mass is required. Such an instability involves either a dissipative factor in order to grow a central mass concentration (Friedli \& Pfenniger 1991; Sellwood, these proceedings), or dynamical friction of dense satellites (Pfenniger 1991, 1992, 1993). In this respect an accretion of $5-10 \%$ of mass of satellites inside the bar region is able to destroy the bar and form a much bigger bulge like the one in M104.

Other indications from $N$-body simulations that the dynamical picture that we propose is essentially correct, are (1) the reshaping of an initial disk into an exponential disk plus a steeper bulge-like profile in the bar region results automatically from the dynamical effects of a bar (Hohl 1971), (2) in such conditions the velocity ellipsoid tends to be anisotropic with an exponential profile consistent with the Milky-Way observations (Lewis \& Freeman 1989): $\sigma_{R}^{2} \propto \exp (-R / h)$, with $\sigma_{R}>\sigma_{\phi}>\sigma_{z}(\mathrm{PF} 91)$

\footnotetext{
${ }^{3}$ In order to explain this surprisingly fierce instability, these authors must finally distinguish it from the fire-hose case and rely on giving an explanation in terms of the "oscillations of stars" with a $2 / 1$ frequency ratio. Of course the orbit description is just the same in a systematic fashion. The reported strong grid dependence in their numerical results seems natural once realized that around a major resonance chaos causes a sensitive dependence not only on the neglected non-linear terms, but also on the numerical technique; this is just the signature of a so-called "ill-posed" problem mentioned above.
} 
All these different kinds of dynamical evolutions give a living character to the spirals, contrasting with older static views. It is then natural to assume that bulges may grow secularly from their disk, which is consistent with the observations that bulge stars are metal rich (Rich 1992). The general picture of disk dynamical evolution is a sequence of barred and unbarred phases through which the bulge size grows irreversibly. This is one of the several arguments for proposing an evolution of the spirals along the Hubble sequence from Sd to Sa (Pfenniger et al. 1994). It is not clear yet how bars can be recurrent, because a central mass concentration once formed should prevent further bar formation unless a large amount of cold and angular momentum rich material is able to accrete quietly on the galaxy disk without heating it.

Finally, a general observed trend concerns the apparent near integrability of stable stellar systems. The phase-space analysis of $N$-body runs, such as in PF91, shows that the final most stable configurations are free of strong chaotic orbits and have remarkably simple phase space structures reminiscent of integrable systems, at least for the populated phase space regions. The remaining ubiquitous weak chaos is indistinguishable from the particle noise. This is consistent with the stability considerations given in Section 4: chaotic regions are likely to seed collective instabilities.

\section{Final Remarks and Conclusions}

The first order dynamics determining the shape and evolution of barred galaxies is now well understood. The component decomposition of these galaxies in bulge, bar, and disk is dynamically illusory since many stars constantly switch from one "component" to the next and back. The gross morphology of bars, their sense of evolution is well understood, and many observed features like the exponential disks, the peanut-shaped bars, and the SB0 profiles can be remarkably reproduced by numerical means. Finer morphologies like rings, ansae, double bars are now at the limit of the numerical resolution in fully self-consistent models.

For understanding galaxies the knowledge of the periodic orbits turns out to be an invaluable tool to simplify the description of the dynamical processes, and to develop an intuition allowing even correct guesses without computer!

Bars can form spontaneously from their disk, peanut-shaped bulges can grow from their parent bar, and bars can fully dissolve in spheroidal bulges through a central accumulation of mass, so secular dynamical evolution of spirals appears as natural, with typical time-scales of the order of $0.1-10 \mathrm{Gyr}$ for changing significantly the spiral type.

Acknowledgments. This work is supported by the Swiss FNRS.

\section{References}

Arnold, V. I. 1989, Mathematical Methods of Classical Mechanics, Springer Verlag

Binney, J. \& Tremaine, S. 1987, Galactic Dynamics, Princeton: Princeton University Press

Combes, F. \& Sanders, R. H. 1981, A\&A, 96, 164 
Combes, F., Debbasch, F., Friedli, D., \& Pfenniger, D. 1990, A\&A, 233, 82

Friedli, D. \& Pfenniger, D. 1990, in Bulges of Galaxies, ESO Conf. and Workshop Proc., B. J. Jarvis, D. M. Tendrup, 265

Friedli, D. \& Pfenniger, D. 1991, in Dynamics of Galaxies and Molecular Clouds Distribution, F. Casoli \& F. Combes, Dordrecht: Kluwer, 362

Hasan, H. \& Norman, C. A. 1990, ApJ, 361, 69

Hasan, H., Pfenniger, D., \& Norman, C. A. 1993, ApJ, 409, 91

Hohl, F. 1971, ApJ, 168, 343

Kennicutt, R. C. 1989, ApJ, 344, 685

Kormendy, J. 1982, in Morphology and Dynamics of Galaxies, L. Martinet \& M. Mayor, Sauverny: Geneva Obs., 113

Leitherer, C. \& Heckman, T. 1995, ApJS, 96, 9

Lewis, J. R. \& Freeman, K. C. 1989, AJ, 97, 139

Merritt, D. \& Sellwood, J. A. 1994, ApJ, 425, 551

Miller, R. H. \& Smith, B. F. 1979, ApJ, 227, 785

Pfenniger, D. 1984, A\&A, 134, 373

Pfenniger, D. 1985, A\&A, 150, 112

Pfenniger, D. 1987, A\&A, 180, 79

Pfenniger, D. 1990, A\&A, 230, 55

Pfenniger, D. 1991, in Dynamics of Disk Galaxies, B. Sundelius, Göteborg: Chalmers Univ. Press, 191

Pfenniger, D. 1992, in Physics of Nearby Galaxies, T. X. Thuan, C. Balkowski, \& J. T. Thanh Van, Ed. Frontières, 527

Pfenniger, D. 1993, in Galactic Bulges, H. Dejonghe \& H. J. Habing, Dordrecht: Kluwer, 387

Pfenniger, D. \& Norman, C. 1990, ApJ, 363, 391

Pfenniger, D. \& Friedli, D. 1991, A\&A, 252, 75 (PF91)

Pfenniger, D., Combes, F., \& Martinet, L. 1994, A\&A, 285, 79

Quirk, W. J. 1972, ApJ, 176, L9

Raha, N., Sellwood, J. A., James, R. A., \& Kahn, F. D. 1991, Nature, 352, 411

Rich, R. M. 1992, in Physics of Nearby Galaxies, T. X. Thuan, C. Balkowski, \& J. T. Thanh Van, Ed. Frontières, 153

Sellwood, J. A. \& Wilkinson, A. 1993, Rep. Progr. Phys., 56, 173

Toomre, A. \& Kalnajs, A. J. 1991, in Dynamics of Disk Galaxies, B. Sundelius, Göteborg: Chalmers Univ. Press, 341

\section{Discussion}

B. Elmegreen: What does a bar look like after it is destroyed? It seems impossible that early type bars can form bulges that big and also that late type bars form bulges, because late type galaxies don't have significant bulges. Perhaps only some S0 galaxies with the largest bulges contain totally destroyed bars. 
D. Pfenniger: In our simulations a fully destroyed bar looks like a spheroid with an extension similar to the original bar. Now I would interpret systems with both bar and bulge as systems right in the process of dissolving the bar; during this stage the bulge size extends up to the ILR, so can be much smaller than the bar such as in late types. In early systems with big bulges, like M104, the size of the bulge is too big to result only from a single bar, from energetics considerations. But our quoted simulations do produce such systems by merging satellites involving in total about $5-10 \%$ of the stellar mass; such merging events also destroy a pre-existing bar.

S. Dodonov: In the 5-Gyr N-body bar the "hot" population showed two distinct bumps in the angular momentum distribution. What are they?

D. Pfenniger: The primary bump is made of the stars trapped by the bar. The secondary bump at higher angular momentum but confined inside corotation consists of particles around the $4 / 1$ and higher order resonances. 